\title{
Photoinactivation of the Crayfish Segmental Giant Neuron Reveals a Direct Giant-Fiber to Fast-Flexor Connection with a Chemical Component
}

\author{
K. Fraser and W. J. Heitler \\ The Gatty Marine Laboratory, Department of Biology and Pre-Clinical Medicine, University of St. Andrews, Fife KY16 8LB, \\ Scotland
}

The escape tail flip of the crayfish is "commanded" by 2 sets of giant-fiber (GF) interneurons. In each hemisegment, these drive the motor giant (MoG) abdominal flexor motorneuron through a monosynaptic electrical connection, but the remaining 8 or 9 fast-flexor (FF) motorneurons receive most of their input via a disynaptic electrical pathway through the segmental giant (SG) neuron. We have investigated a monosynaptic GF-FF pathway, which operates in parallel to the disynaptic GF-SG-FF pathway, by using dye-mediated photoinactivation to remove the SGs from the tail-flip circuit.

SG photoinactivation involves an initial broadening of the spike, leading to a long-duration, massively depolarized plateau. This is followed by loss of spike capability, a gradual reduction in the resting potential, and eventual total loss of electrical responsiveness. After bilateral photoinactivation of the SGs, a spike in one set of GFs, the medial giants (MGs), produces little if any effect in FFs in any ganglion. A spike in the other set, the lateral giants (LGs), produces an EPSP in FFs with a declining anterior-to-posterior segmental gradient in amplitude. These differences in LG and MG outputs, which are obscured in the intact circuit by the common MG/LG-SG-FF pathway, give clues to a probable early evolutionary form of the circuit.

The LG-FF connection in anterior ganglia has a significant electrical component. However, it also has an apparent monosynaptic chemical component, as revealed by the response to saline containing cadmium ions, and to cooling the preparation. This is the first physiological evidence for chemical output from a crayfish GF.

The escape tail flip of the crayfish is one of the most studied and best understood neural circuits in the entire animal kingdom (see, e.g., Wine and Krasne, 1982; Wine, 1984 for reviews). The central feature of the circuit is the 2 sets of giant "command" fibers (GFs), which run the length of the animal. A spike in one of the GFs is sufficient to initiate the flexion phase of the tail flip (Wiersma, 1947). However, there is a puzzling feature in the output circuitry of these GFs. In each hemisegment, they drive one of the abdominal flexor motorneurons, the motor giant

\footnotetext{
Received May 4, 1990; revised Aug. 14, 1990; accepted Aug. 30, 1990.

This work was supported by a grant to W.J.H. from the Science and Engineering Research Council of the United Kingdom.

Correspondence should be addressed to Dr. W. J. Heitler, The Gatty Marine Laboratory, Department of Biology, University of St. Andrews, Fife KY16 8LB, Scotland.

Copyright (C) 1991 Society for Neuroscience $0270-6474 / 91 / 010059-13 \$ 03.00 / 0$
}

(MoG), directly through powerful 1:1 rectifying electrical synapses (Furshpan and Potter, 1959a), but the remaining 8 or 9 nongiant fast-flexor (FF) motorneurons receive most of their input from the GFs via an intermediary neuron called the segmental giant (SG; Kramer et al., 1981; Roberts et al., 1982). The SG is an enigmatic neuron: it has the central anatomy of a limb motorneuron and an axon in the nerve root (the first root; R1) innervating the limb, but this axon is blind-ending and has no known function (Heitler and Darrig, 1986; Fraser and Heitler, 1989). The SG thus acts as a relay interneuron, receiving 1:1 input from the GFs through rectifying electrical synapses and making output to the FF motorneurons, also through rectifying electrical synapses. The puzzling feature referred to above is why the SG, with the apparent provenance of a limb motorneuron, occurs in the middle of a circuit concerned with tail flexion.

The abdominal neurons that receive major direct input from the GFs, the MoG and the SG, are clearly highly specialized neurons that have been fine tuned by evolution for their role in the escape tail flip. In contrast, the FF motorneurons, from which the MoGs evolved, are rather unspecialized neurons. They are neither giant [compared to the lateral giant (LG), medial giant (MG), MoG, and SG] nor enigmatic (they have normal functional axons, in contrast to the $\mathrm{SG}$ ). The major input to the FFs that occurs when a GF spikes is undoubtedly disynaptic, mediated through the SGs. However, by subtracting the FF response to antidromic $\mathrm{SG}$ activation from the response to $\mathrm{GF}$ activation (which, of course, includes the SG-mediated response), it appears that there may be some direct activation of the FFs by the GFs. This has been estimated to be between 0 and $5 \%$ of the total input, in different preparations (Roberts et al., 1982). The aim of the present paper is to characterize this possible direct GF-FF interaction in detail. This has been achieved by filling the SG with the fluorescent dye Lucifer yellow (Stewart, 1978), then photoinactivating it (Miller and Selverston, 1979). When the major input to the FFs from the SG has been eliminated, any remaining component from the GFs is more easily studied. The interest of the direct GF-FF connection is that it may reveal the primitive, baseline characteristics of the relationship between the GFs that drive the tail flexion and the FFs that must have been the original motorneurons that implemented it. Particular questions of interest include the following.

Is there really a direct input to the FFs from the GFs? The subtraction studies are suggestive but not conclusive, owing to the small size of the putative direct input, the existence of other 
possible paths for activating the FFs (the corollary discharge interneurons; e.g., Kramer et al., 1981), and the small but definite inherent variability of the responses.

Are there differences between the GFs? One set of GFs is comprised of the bilaterally paired MG neurons. These are activated by anterior stimuli and drive all the abdominal MoGs, causing a uniform abdominal flexion and backwards movement. The other set of GFs is the LGs. These are activated by posterior stimuli and drive the MoGs only in the anterior part of the abdomen (Mittenthal and Wine, 1978a). This causes an abdominal flexion with a "jack-knife" form, and a forward somersault. Because both the LG and the MG activate the SG in every segment, the final common SG-FF path ensures that there is no significant difference between activation of the FFs by the LG and by the MG. However, there are segmental differences between the $L G$ and the MG in terms of their direct output to the MoG, so there might also be differences in any direct output they make to the FFs.

Are there differences between segments in any direct output from the GFs to the FFs? There is a segmental gradient in the output from the SG to the FFs, such that SGs in anterior segments make more powerful output than SGs in posterior segments (Miller et al., 1985). Does a similar gradient occur in the direct GF-FF connection?

What is the mechanism of any direct output from the GFs to the FFs? In particular, is the output through electrical synapses (and if so, are they rectifying), or is the output through chemical synapses, or is it mixed?

\section{Materials and Methods}

Experiments were performed on the CNS of the crayfish Pacifastacus leniusculus. Animals were obtained from Riversdale Farm (Stour Provost, Near Gillingham, Dorset, UK). The nerve cord was dissected as follows. An animal was anesthetized by cooling on ice for $20 \mathrm{~min}$, then decapitated. The legs and chelae were severed at the autotomy plane. The dorsal carapace and viscera were removed, and the nerve cord was dissected from the animal. The last 2 thoracic ganglia and the entire abdominal chain of ganglia were removed. The nervous tissue was then pinned dorsal surface upwards on a Sylgard platform and submerged in Van Harreveld's crayfish saline.

Bipolar hook electrodes were used to record extracellularly and to stimulate the connectives. Pin electrodes were used to record extracellularly and to stimulate the roots, with a paired indifferent electrode for each pin placed adjacent to it in the preparation bath. Intracellular recordings were made with glass microelectrodes (resistance, $15-40 \mathrm{M} \Omega$ ) filled either with $5 \%$ Lucifer yellow dissolved in $1 \mathrm{~mol} /$ liter lithium chloride or with $2 \mathrm{~mol} / \mathrm{liter}$ potassium acetate. Penetrations were all made from the dorsal aspect of the ganglion into axonal or neuropil processes. The ganglionic sheath was removed prior to penetration. In all records showing the voltage response of a neuron to current injection, separate current-passing and voltage-recording electrodes were used. Current was measured with a virtual ground current monitor. The output feedback from this circuit (i.e., the nulling current) was fed back into the preparation bath to minimize artifactual voltage coupling between current-passing and voltage-recording electrodes (Purves, 1981).

Neurons were injected with Lucifer yellow using 0.5 -sec negative current pulses of 10-20 nA delivered at $1 \mathrm{~Hz}$ for up to $1 \mathrm{hr}$. Dye visualization and photoinactivation were achieved using a liquid light guide attached to an epifluorescent compound microscope (Heitler and Fraser, 1989). Following photoinactivation, in many experiments, a single ganglion was isolated by cutting the anterior and posterior connectives.

\section{Results}

Characteristics of SG photoinactivation

The characteristics of a simple photoinactivation experiment are described first (Fig. 1). In this experiment, simultaneous microelectrode penetrations of an FF motorneuron, the ipsilateral SG (iSG), and the LG were made, and the SG was then filled iontophoretically with Lucifer yellow. No dye coupling between the SG and other neurons was observed in this or any other experiments. Once an adequate stain was achieved, as judged by very brief viewing with epi-illumination by unfocused blue light, the basic characteristics of the intact system were determined.

Extracellular stimulation of the anterior connective (AC) activates both the GFs and the SGs (via the GFs) and drives a large EPSP in the FF (Fig. 1A, i). Extracellular stimulation of the ipsilateral first root (iR1) activates the iSG (but not the LG) and drives an EPSP in the FF that is slightly smaller than that initiated by $\mathrm{AC}$ stimulation (Fig. $1 \mathrm{~A}$, ii). Extracellular stimulation of the contralateral first root (cR1) activates the contralateral SG (cSG) and drives a small EPSP in the iSG and a small EPSP in the FF (Fig. 1A, iii).

Photoinactivation was initiated by exposing the ganglion containing the stained SG to a focused beam of intense blue light. The AC was stimulated repetitively at $0.5 \mathrm{~Hz}$ to monitor physiological changes in the system while photoinactivation progressed. After 5-10 min, the SG started to depolarize, and its spike to broaden, in response to AC stimulation. This was accompanied by an increase in amplitude of the EPSP in the FF and the appearance of an obvious step on its rising phase (Fig. $1 B$, i). A few minutes later, the SG was depolarized by almost $30 \mathrm{mV}$, its spike had a clear plateau on its falling phase, and the FF EPSP was now large enough to cause the FF to spike (Fig. 1B, ii). With continued illumination, the SG plateau increased in duration, with a fully depolarized level of almost 80 $\mathrm{mV}$ above rest. This caused a prolonged EPSP in the FF, with multiple spikes (Fig. $1 B$, iii). At this point, the FF had a resting potential that was depolarized by about $10 \mathrm{mV}$ relative to its value at the start of the experiment. After about $25 \mathrm{~min}$, the spike in the SG started to fail, revealing the underlying EPSP caused by the AC stimulation. As the SG spike failed, the large EPSP in the FF was replaced by a much smaller potential (Fig. $1 B$, iv). After nearly $1 \mathrm{hr}$ of illumination, the $\mathrm{SG}$ was depolarized by more than $60 \mathrm{mV}$ and reccived only a small potential in response to AC stimulation (Fig. $1 B$, v). After a further $10 \mathrm{~min}$, the FF resting potential had returned to its approximate level at the start of the experiment, and the SG ceased to show any response to $\mathrm{AC}$ stimulation (Fig. $1 \mathrm{~B}$, vi). At this point, it was depolarized by $88 \mathrm{mV}$ relative to its initial resting potential, and withdrawal of the microelectrode showed that it had completely lost its resting membrane potential. It was thus regarded as dead.

After photoinactivating the iSG, the characteristics of the remaining input to the $\mathrm{FF}$ were determined by extracellular stimulation of the $A C$ and the $\mathrm{CR} 1$. In this particular experiment, $\mathrm{AC}$ stimulation yielded a potential that was of similar amplitude, but longer in duration, than that caused by $\mathrm{cR} 1$ stimulation. Throughout the experiment, the LG response to AC stimulation was checked periodically and showed no change.

\section{Photoinactivation with an intact abdominal nerve cord}

Because the FFs receive input from both the iSG and the $\mathrm{CSG}$, clearly, the next requirement was to photoinactivate both SGs. This was achieved (Fig. 2) over a similar time course, and with similar characteristics, to the experiment described above (Fig. 1). After about $1 \mathrm{hr}$ of illumination, both SGs had lost all resting potential and all sign of input resulting from AC stimulation. 

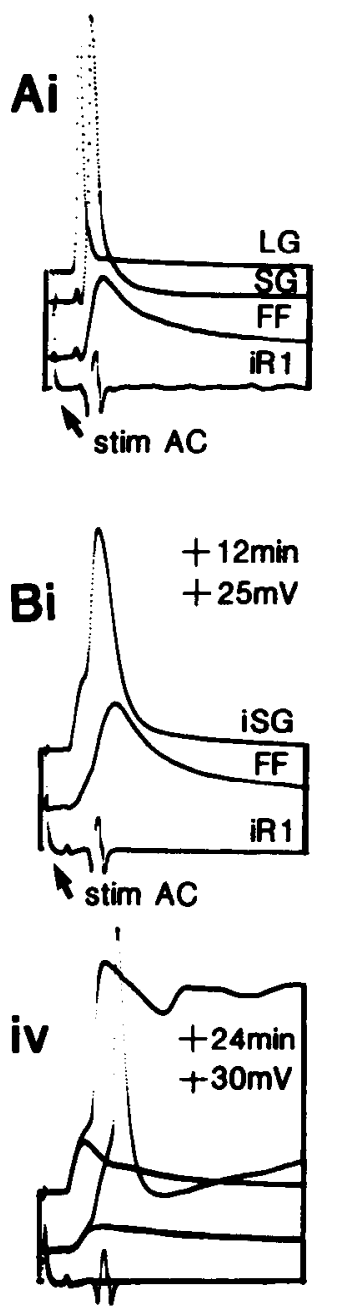
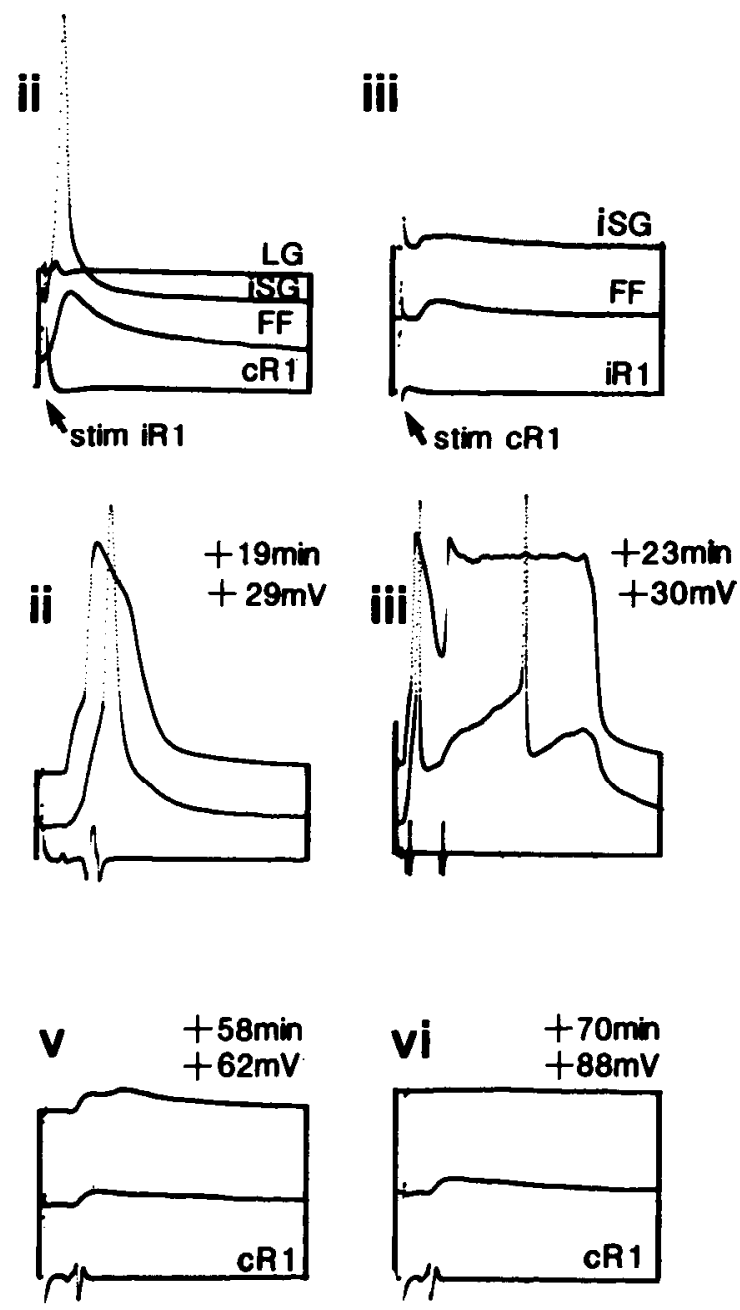

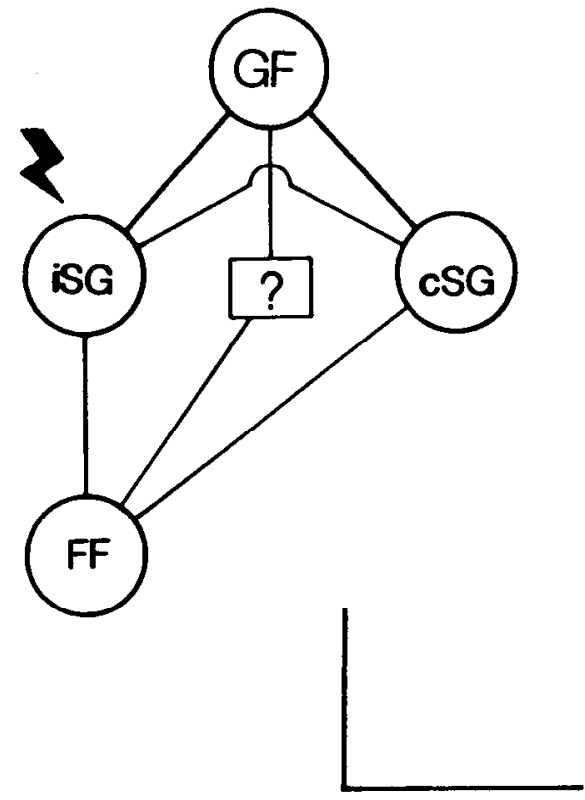

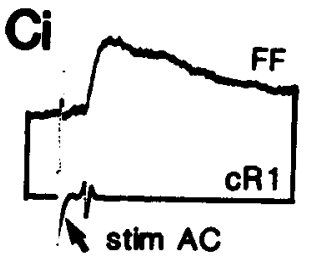

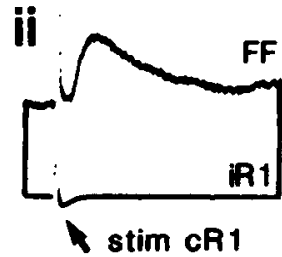

Figure 1. General characteristics of photoinactivation experiment. $A$, Properties prior to photoinactivation. $i$, Stimulating the AC initiates a spike in the LG (1st trace) and the iSG (2nd trace), and an EPSP in the FF (3rd trace). The axon spike of the SG is recorded extracellularly in iR 1 (4th trace). $i$, Stimulating the axon of the iSG within iR 1 initiates an antidromic spike in the iSG and an EPSP in the FF (traces as in $i$ ). No spike is recorded in the cRI (4th trace). iii. Stimulating the axon of the cSG within cRl elicits an EPSP in the iSG (1st trace) and the FF (2nd trace). No spike is recorded in the $\mathrm{iR} 1$ ( $3 \mathrm{rd}$ trace). $B$, Time course of photoinactivation, with the AC continuously stimulated at $0.5 \mathrm{~Hz}$. The elapsed time from the start of photoinactivation, and the SG membrane potential relative to its initial value, are given on each record. $i$, The iSG has depolarized, and its spike has broadened (1st trace). The EPSP in the FF has increased in amplitude (2nd trace). The iSG axon spike is recorded extracellularly in iR1 (3rd trace). ii, The SG spike has a distinct plateau on its falling phase, and the FF EPSP now initiates a spike. iii, The SG has a long depolarized plateau, and 2 axon spikes are recorded in $\mathrm{RR} 1$. The extended EPSP in the FF initiates 2 spikes. Note the longer timebase of record. $i v$, The SG spike starts to fail. Records from 2 consecutive AC stimuli are superimposed. Note that the failure of the SG axon spike is coincident with failure of the SG central spike. $v$. There is now little response in the SG. The axon spike of the cSG recorded in cR1 is now displayed (3rd trace). vi, The iSG is dead. $C$, Properties following iSG photoinactivation. $i$, Stimulating the AC elicits a relatively small EPSP in the FF (Ist trace) and a spike in the cSG recorded in cR1 (2nd trace). $i$. Stimulating the axon of the cSG in cRl elicits a small EPSP in the FF (Ist trace). No spikes are recorded in the $\mathrm{iRI}$ (2nd trace). The diagram above shows the experimental arrangement, with the GF at the top, the bilateral pair of SGs on either side, and the FF below. Calibration bars: Vertical-LG, $60 \mathrm{mV} ; S G(A, i, i i ; B), 60 \mathrm{mV} ; S G(A, i i i), 30 \mathrm{mV} ; F F(A, B), 30 \mathrm{mV} ; F F(C), 6$ $\mathrm{mV}$. Horizontal-A; B, i, ii, iv-vi; $C, 20 \mathrm{msec} ; B, i i i, 80 \mathrm{msec}$.

However, both AC stimulation and direct stimulation of the LG with injected positive current revealed a relatively large, multicomponent EPSP in the FF (Fig. 2C). The initial phase had a short latency and sharp rise time, suggesting that it might be a monosynaptic response to the LG spike, but it was clearly contaminated by later, polysynaptic components. These probably result from input to the FFs from corollary discharge interneurons (CDIs) activated by the GFs, including the identified CDIs I2 and I3, which are activated via the SGs of other ganglia. Thus, in order to eliminate all known paths exciting the FFs except the putative direct path from the GFs, it is necessary to work on a single isolated ganglion in which both SGs have been photoinactivated.

\section{Photoinactivation of both SGs in isolated ganglia}

The first ganglion (G1) was isolated from the rest of the abdominal nervous system by severing the connectives. The CSG and iSG were filled sequentially with Lucifer yellow through intracellular iontophoresis, then a microelectrode was placed in an FF motorneuron, and 2 microelectrodes were placed in the LG. The filling electrode was maintained in the iSG. This enabled us to monitor the LG-iSG-FF path by injecting positive current 

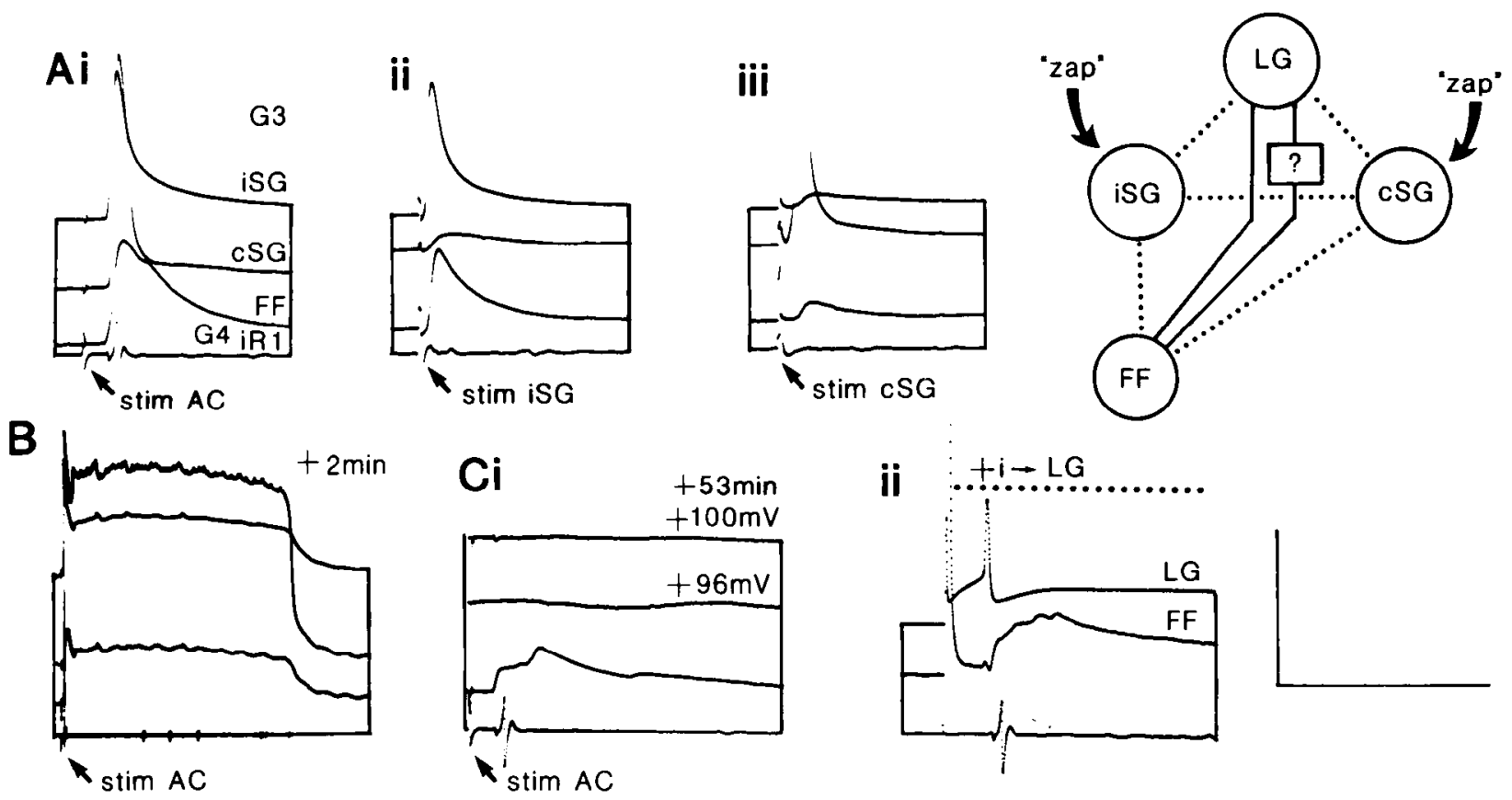

Figure 2. Photoinactivation of bilateral pair of SGs in third ganglion (G3) with abdominal nerve cord intact. $A$, Properties prior to photoinactivation. $i$. Stimulating the AC initiates a spike in the iSG (1st trace) and the CSG (2nd trace), and a large EPSP in the FF ( $3 r d$ trace). The axon spike of the iSG in the fourth ganglion (G4) is recorded in the iR1 of G4 (4th trace). ii, Stimulating the axon of the iSG in iR1 initiates an antidromic spike in the iSG, a small EPSP in the cSG, and a large EPSP in the FF. iii, Stimulating the axon of the cSG in cR1 initiates a small EPSP in the iSG, an antidromic spike in the CSG, and a small EPSP in the FF. B. Partial photoinactivation. Stimulating the AC initiates spikes in both SGs with extended plateau phases. $C$, Properties following photoinactivation. $i$, Stimulating the AC has no effect on either SG, but produces a multicomponent EPSP in the FF. ii, Positive current injected into the LG (dotted line above record indicates pulse duration) elicits a spike (1st trace) and a multicomponent EPSP in the FF (2nd trace). Calibration bars: Vertical-SG, $60 \mathrm{mV} ; F F, 30 \mathrm{mV} ; L G, 150 \mathrm{mV}$. Horizontal-A and $C, 20 \mathrm{msec}$; $B, 200 \mathrm{msec}$.

into the LG (Fig. 3A, i), and the iSG-FF and cSG-FF paths by extracellular stimulation of the iSG axon through iR 1 (Fig. $3 A$, ii) and the cSG axon through cR1 (Fig. $3 A$, iii). Photoinactivation was then commenced. We could follow the course of $\mathrm{iSG}$ inactivation (Fig. $3 B$ ) with the intracellular recording, but because we were unable to place more than 4 microelectrodes simultaneously within the ganglion, the cSG inactivation had to be estimated from previous experience of the time course (Figs. 1, 2) and from the timing of the failure of the CSG spike recorded extracellularly in $\mathrm{cR} 1$. When both SGs were dead, the LG-FF path was tested by driving the LG with injected current. This revealed an EPSP in the FF of about $5 \mathrm{mV}$ amplitude (Fig. $3 C$, i). The latency from the LG spike to the EPSP was very short (less than $0.2 \mathrm{msec}$; Fig. $3 C$, ii), suggesting that the interaction was monosynaptic and electrical (but see below). Subthreshold positive current injected into the LG spread to the FF (Fig. 3C, iii). There was also some spread of hyperpolarizing current from the LG to the FF, but this was considerably less than that of depolarizing current. The voltage-coupling ratio from the LG to the FF for subthreshold current in the depolarizing direction was approximately 10:1.

\section{Comparison of direct output from LG and MG to FFs}

In the intact animal, the majority of the input to the FFs that occurs when the GFs spike is routed via the SGs, and the LG and the MG both make suprathreshold input to the SGs in every ganglion. This means that there is very little difference in the FF input that occurs when either the MG or the LG spikes in the intact nervous system. However, there is a major difference between the direct input to FFs from the LG and the MG fol- lowing photoinactivation of the 2 SGs (Fig. 4). The direct input to an FF in Gl when the LG is specifically activated by current injection can be as large as $12 \mathrm{mV}$. However, the largest input seen in the same FF when the MG was specifically activated by current injection was $0.5 \mathrm{mV}$ (Fig. $4 A$, i, ii), and in many cases, no input was apparent at all.

\section{Comparison of direct input to FFs in first and last ganglia}

We compared the input received by $3 \mathrm{FF}$ motorneurons before and after photoinactivation of the SGs in the isolated first and last free abdominal ganglia (G1 and G5). Prior to photoinactivation, we tested the FF response to 4 stimulation regimes: (1) extracellular stimulation of the AC, (2) intracellular stimulation of the LG with injectcd current, (3) extracellular stimulation of iR 1 to drive the iSG, and (4) extracellular stimulation of $\mathrm{CR} 1$ to drive the $\mathrm{CSG}$. Following photoinactivation of the SGs, we tested the FF response to intracellular stimulation of the LG alone (Table 1). The results showed a considerable difference between the direct LG-FF input at the opposite ends of the abdominal nerve cord. In $\mathrm{G} 1$, the direct input from the LG alone could be as high as $25 \%$ of that mediated by the LG plus the 2 SGs in the intact system (e.g., Fig. 3). In G5, the SG makes a significant input to the FFs (Fig. $5 A$ ), though this is smaller than in anterior ganglia (Miller et al., 1985), but there is no apparent direct input at all (Fig. 5C). There is thus a significant segmental gradient from anterior to posterior in the strength of the direct input from the LGs to the FFs. Further data from FFs examined only after SG photoinactivation suggests that this gradient is fairly smooth across the chain of ganglia (see next section). 

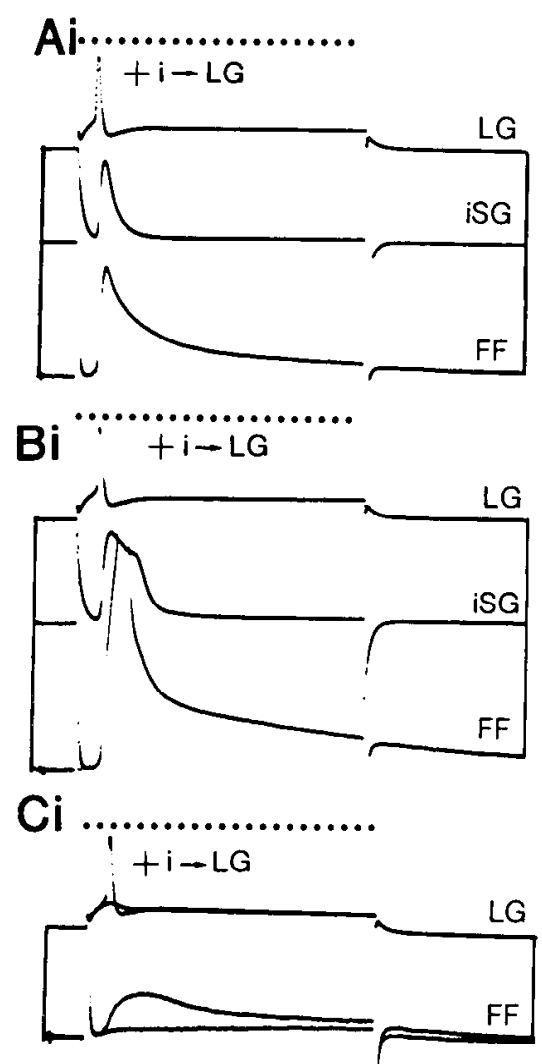
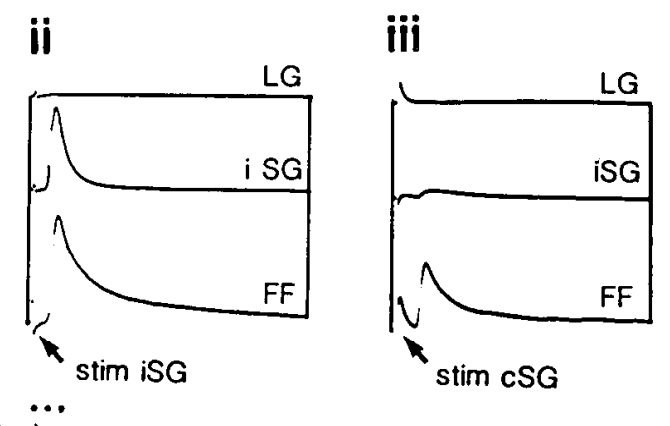

ii

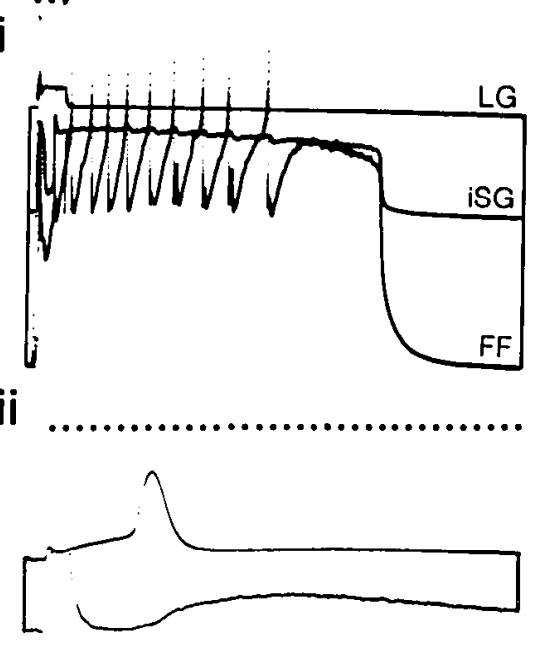

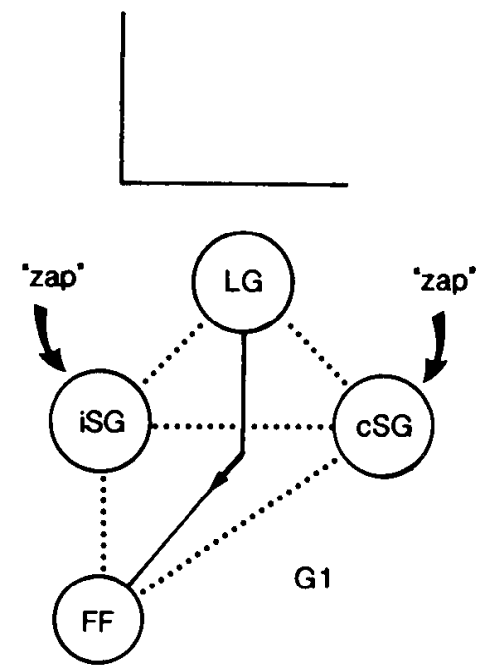

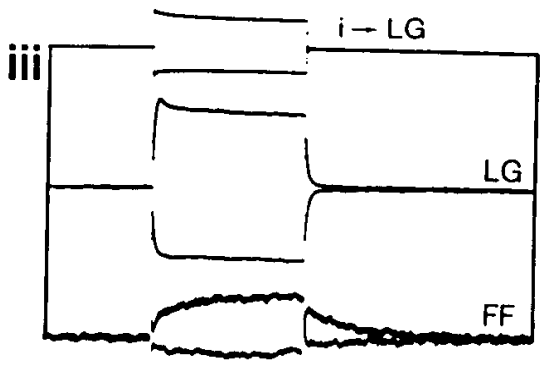

Figure 3. Photoinactivation of bilateral pair of SGs in isolated G1. A, Properties prior to photoinactivation. $i$, Positive current (dotted line) injected into the LG (1st trace) elicits a spike in the iSG (2nd trace) and a large EPSP in the FF (3rd trace). ii. Stimulating the iSG axon in iR 1 elicits an antidromic iSG spike and a large EPSP in the FF. iii, Stimulating the cSG axon in cR 1 elicits a small EPSP in the iSG and FF. $B$, Partial photoinactivation. $i$, The spike in the iSG is starting to broaden in response to a current-initiated spike in the LG. The FF EPSP is now suprathreshold. $i i$, The iSG spike is now massively prolonged, and multiple spikes occur on the FF EPSP. Note the longer timebase of the record. $C$, Properties following bilateral SG photoinactivation. $i$, Two positive current pulses were injected into the LG (Ist trace), one above and one below threshold. The suprathreshold pulse initiates an EPSP in the FF (2nd trace). ii, Fast timebase recording shows short latency between LG spike and FF EPSP. iii. Two superimposed sweeps of positive and negative current pulses (1st trace monitor) were injected into the LG (2nd trace), showing propagation of the voltage perturbation to the FF (3rd trace). Calibration bars: Vertical $-L G(A ; B ; C, i, i i), 150 \mathrm{mV} ; L G(C, i i i), 30 \mathrm{mV} ; S G, 150 \mathrm{mV} ; F F(A$, B), $30 \mathrm{mV} ; F F(C, i, i i), 15 \mathrm{mV} ; F F(C, i i i), 6 \mathrm{mV}$. Current, $300 \mathrm{nA}$. Horizontal $-A ; B, i ; C, i, 20 \mathrm{msec} B, i i, 200 \mathrm{msec} ; C, i i, 4 \mathrm{msec} ; C$, iii, 40 msec.

\section{Comparison of direct input to identified FFs of different classes}

There are 3 anatomical classes of FF motorneuron: FPI, FMC, and FAC (Selverston and Remler, 1972; Mittenthal and Wine, 1978b), and we wished to determine whether there was any consistent difference in the direct input received by members of these different classes. We consistently failed to achieve adequate staining using conventional cobalt techniques with microelectrodes, and instead relied exclusively on Lucifer yellow. This caused a problem: If we penetrated the FF with a Luciferfilled electrode prior to photoinactivating the SGs in order to measure its input in the intact system, we invariably found that the FF itself filled with dye and started to photoinactivate while we were photoinactivating the SGs. This happened even when a positive retaining current of several nanoamps was applied. We therefore had to penetrate the FF after we had fully photoinactivated the SGs. This meant that we could only assess the direct input from GFs after photoinactivation and could not compare this with the total normal input in the intact ganglion.

The results fully confirmed the segmental differences and the differences in LG-mediated and MG-mediated direct input described above (Table 2). However, we noted no consistent differences between the levels of direct input received by FFs of the 3 different anatomical classes.

\section{Comparison of ipsilateral and contralateral input from SGs to FFs}

If the intensity of dye in the 2 SGs was not equal, we usually found that the time course of photoinactivation was unequal, also, with the less intensely stained SG taking longer to inactivate. This enabled us to visualize the relative inputs to a particular FF from the ipsi- and contralateral SGs, as well as the degree of bilateral coupling between the 2 SGs themselves. In one experiment (Fig. 6), the SG ipsilateral to an FF was stained more intensely than the contralateral SG. Shortly after photoinactivation commenced, the iSG spike developed a prolonged plateau, while the cSG was still spiking normally (Fig. $6 B$, i). This plateau propagated to the $\mathrm{FF}$ as a large depolarizing potential. It also propagated to the cSG through the bilateralcoupling electrical synapse, causing a prolonged depolarization. This was small compared to the plateau in the iSG, but none- 

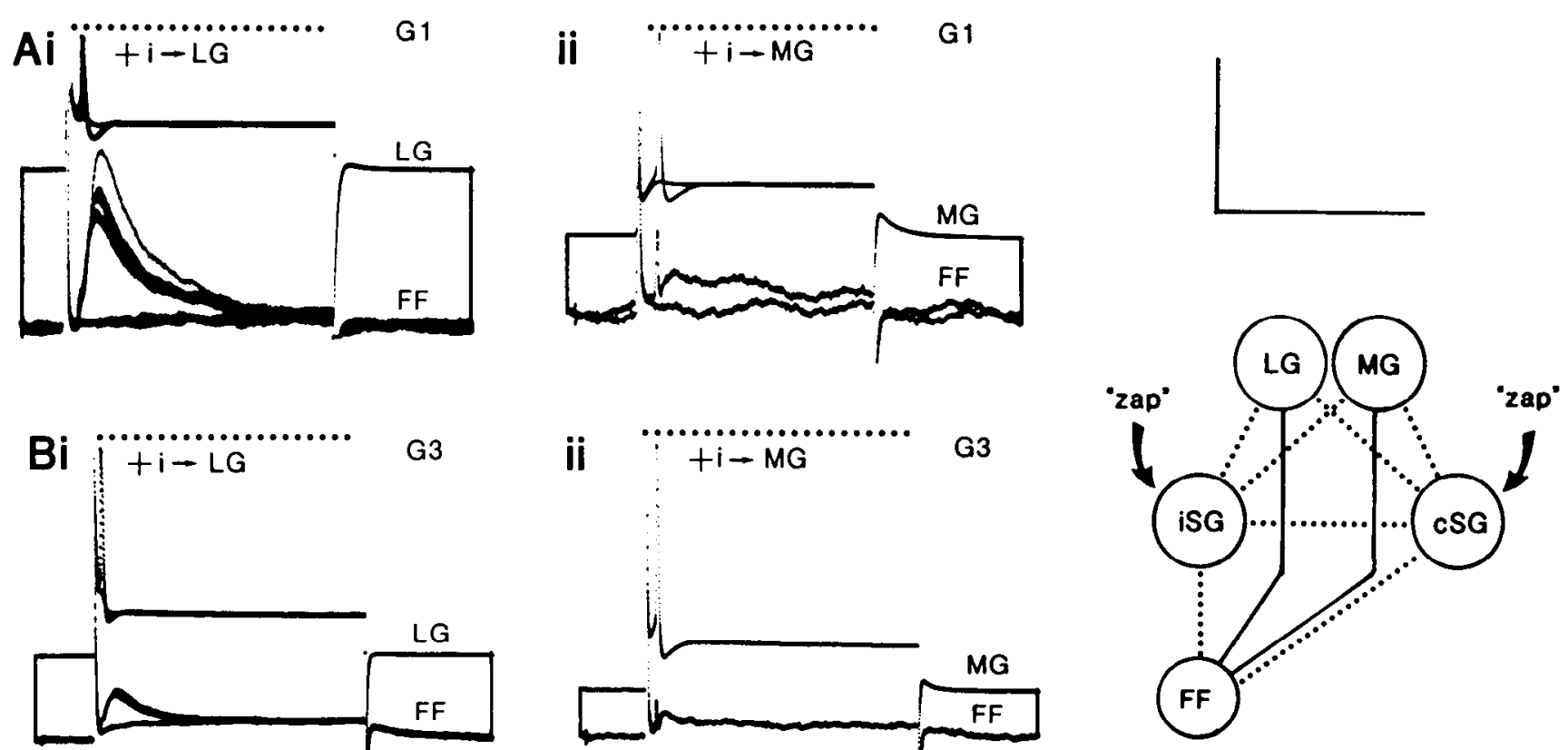

Figure 4. Comparison of input to FFs from LG and MG after bilateral photoinactivation of SGs in isolated ganglion. $A$, $i$, Several superimposed records show positive current (dotted line) injected into the LG (1st trace) at $1 \mathrm{~Hz}$. In the first few records, the current was just subthreshold. It was then increased fractionally and became suprathreshold. When the LG spiked, it elicited an EPSP in an FF in Gl (2nd trace). Note the decrement in the EPSP amplitude with repeated LG spikes. $A$, $i$, A spike in the MG (1st trace) produces little if any output to the same FF (2nd trace). $B, i$, Another FF recorded in $G 3$ receives input from the LG. $B$, ii, This FF also receives little if any input from the MG. Calibration bars: Vertical$L G$ and $M G, 60 \mathrm{mV} ; F F(A ; B, i i), 6 \mathrm{mV} ; F F(B, i), 15 \mathrm{mV}$. IIorizontal-40 msec.

theless generated several cSG spikes. These induced phasic EPSPs on the prolonged EPSP in the FF (Fig. 6B, i). After a further period of photoinactivation, the iSG lost its spike, while the cSG spike started to broaden. At this point, the input to the FF was greatly reduced (Fig. $6 B$, ii). Finally, the cSG spike also developed the prolonged plateau, and this propagated to the FF as a series of small potentials (Fig. 6B, iii). The iSG was almost completely inactivated, and only a very small prolonged depolarization occurred in it.

\section{Chemical component to the direct GF-FF interaction}

When the SGs have both been photoinactivated, subthreshold current of either polarity injected into the LG in Gl produces a small voltage shift in an FF (Fig. $3 C$, iii), with depolarizing current spreading preferentially compared to hyperpolarizing current. This, coupled to the short latency with which the FF EPSP follows an LG spike (Fig. $3 C$, ii), suggests that the direct output from the LGs to the FFs is mediated, at least in part, by an electrical synapse. However, a striking feature of the EPSP induced in the FFs by the LG is that it often shows pronounced decrement with repeated activation of the LG, even at relatively low frequency (e.g., Fig. $4 A$, i). Decrement of this type is commonly associated with a chemical synapse. It is by no means conclusive evidence of a chemical component to a synaptic interaction, because it can be produced by purely postsynaptic mechanisms (Fraser and Heitler, 1987), but it provided an initial indication to us that there might be a chemical component to the direct LG-FF interaction.

We have applied 2 experimental procedures to try to determine whether there is indeed a chemical component to the direct GF-FF interaction. The first was to apply cadmium ions to the system. This should block calcium channels (Kostyuk et al.,
1977) and therefore prevent the presynaptic release of transmitter. The second was to cool the preparation. This should increase the latency of a chemical synapse more than that of an electrical synapse and therefore may produce a discontinuity on the rising phase of the EPSP.

\section{Effects of cadmium ions}

Two initial control experiments were required, first to demonstrate that cadmium really does block chemical synapses, and

Table 1. Amplitude of EPSPs (mV) in FF motorneurons in G1 and G5 before and after bilateral photoinactivation of the SGs

\begin{tabular}{|c|c|c|c|c|c|}
\hline & Befor & otoina & tion & & $\begin{array}{l}\text { After } \\
\text { photo- } \\
\text { inacti- } \\
\text { vation: }\end{array}$ \\
\hline & $\mathrm{AC}$ & LG & iR1 & cR 1 & LG \\
\hline G1 & 19.5 & 19.5 & 19 & 11.0 & 3.0 \\
\hline & 27.0 & 23.5 & 21.5 & 10.5 & 3.75 \\
\hline & 22.5 & 18.0 & 15.5 & 1.5 & 5.5 \\
\hline G5 & 14.0 & 13.5 & 11.5 & - & 0.0 \\
\hline & 22.5 & 20.5 & 18.5 & 5.0 & 0.5 \\
\hline & 10.0 & 9.0 & 7.0 & 3.0 & 0.0 \\
\hline
\end{tabular}

Extracellular stimuli were applied to the $\mathrm{AC}$, $\mathrm{RR} 1$, and $\mathrm{CR} 1$. The stimulus strength was the minimum required to elicit a spike in the LG, iSG, and CSG, respectively. Suprathreshold positive current was injected into the LG. Recordings were made from FF motorneurons using microelectrodes filled with potassium acetate; the class of the individual FFs was therefore unknown (see Results). The - indicates data not available. Note that the amplitude of the FF EPSP due to direct LG stimulation is always greater than that caused by stimulating either the iSG or the cSG, but is less than the sum of those EPSPs. We did not attempt simultaneous stimulation of the 2 SGs. 

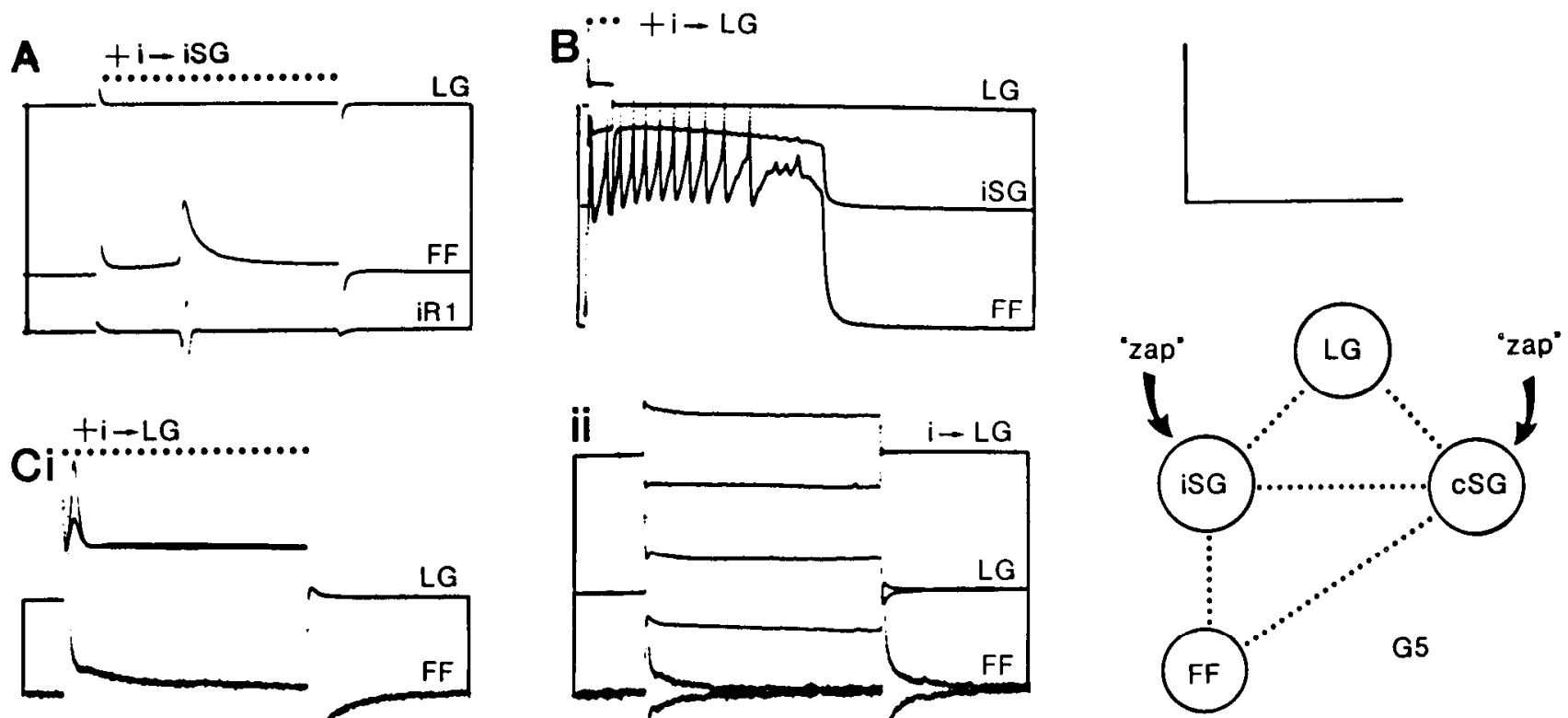

Figure 5. FFs in G5 receive no input from LG. $A$, Prior to photoinactivation. Positive current (dotted line) injected into the iSG (not shown) has no effect on the LG (1st trace) and produces an EPSP in an FF (2nd trace). The axon spike of the iSG is recorded in the iR 1 (3rd trace). $B$, Partial photoinactivation of the iSG. Positive current injected into the LG (1st trace) initiates a spike, which causes a massively prolonged spike in the iSG (2nd trace). Note that this has no effect on the LG, but causes a prolonged EPSP with multiple spikes in the FF (3rd trace). $C$, Properties following bilateral photoinactivation of the SGs. $i$, Sub- and suprathreshold positive current injected into the LG (1st trace) has no difference in effect on the FF (2nd trace). $i$, Positive and negative current (1st trace monitor) injected into the LG (2nd trace) does not propagate to the FF ( $3 r d$ trace). The switching transient is accentuated at the high gain. Calibration bars: Vertical $-L G(A, B), 150 \mathrm{mV} ; L G(C), 60 \mathrm{mV} ; S G, 150 \mathrm{mV} ; F F$ $(A, B), 30 \mathrm{mV} ; F F(C), 6 \mathrm{mV}$. Horizontal-A; $C, i, 20 \mathrm{msec} ; B, 200 \mathrm{msec}, C, i i, 40 \mathrm{msec}$

\begin{tabular}{|c|c|c|c|}
\hline Ganglion & FF class & LG stimulus & $\begin{array}{l}\text { MG } \\
\text { stimulus }\end{array}$ \\
\hline \multirow[t]{8}{*}{ G1 } & FPI & 10.0 & - \\
\hline & & 4.0 & - \\
\hline & & 12.0 & - \\
\hline & & 8.0 & - \\
\hline & & 1.2 & - \\
\hline & FMC & 2.0 & - \\
\hline & & 4.7 & - \\
\hline & FAC & 6.7 & 0.5 \\
\hline \multirow[t]{2}{*}{$\mathrm{G} 2$} & FPI & 4.5 & - \\
\hline & FMC & 2.0 & 0.0 \\
\hline \multirow[t]{5}{*}{ G3 } & FPI & 6.0 & - \\
\hline & & 1.2 & - \\
\hline & & 3.5 & 0.5 \\
\hline & FMC & 2.2 & - \\
\hline & FAC & 2.0 & 0.5 \\
\hline \multirow[t]{6}{*}{ G5 } & FPI & 1.0 & 0.0 \\
\hline & & 0.0 & 0.0 \\
\hline & & 0.0 & - \\
\hline & & 0.0 & - \\
\hline & & 0.0 & - \\
\hline & FAC & 0.0 & - \\
\hline
\end{tabular}

The EPSPs were elicited by suprathreshold current pulses injected into the LG and MG. Recordings were made from FF motorneurons using microelectrodes filled with Lucifer yellow in order to achieve anatomical classification; the amplitudes of the EPSPs prior to SG photoinactivation are therefore unknown (see Results). A - indicates data not available. second, to determine whether cadmium has any effects on known electrical synapses. These could be accomplished in a single type of preparation. The GF-MoG interaction is a classic example of an electrical synapse (Furshpan and Potter, 1959a), but the MoG also receives large chemical synaptic potentials [depolarizing IPSPs (dIPSPs); Furshpan and Potter, 1959b; Ochi, 1969]. Ultrastructural examination shows that the sites of chemical synaptic input are very close to the sites of the electrical synapse, and thus it is likely that cadmium has equal access to the 2 types (Stirling, 1972; Leitch et al., 1990). Some of the interneurons eliciting the dIPSPs are intersegmental and have axons that can be directly stimulated in the AC (Wine, 1977; K. Fraser and W. J. Heitler, unpublished observations).

Cadmium ions at $2 \mathrm{~mm}$ concentration totally block the chemical input onto the MoG and the increase in postsynaptic conductance that this input normally produces (Fig. 7). However, cadmium does not block the 1:1 transmission of spikes from the GF to the MoG. In fact, when the MoG response is subthreshold, a frequent effect of cadmium is to increase the size of the GF-induced electrical EPSP, often taking it above threshold (Fig. 8). This is probably due to broadening of the presynaptic GF spike, which is in turn probably due to abolition of a calcium-induced potassium current, though this has not been explicitly tested. Cadmium has no consistent effect on the membrane input impedances of the GF or the MoG, or the subthreshold coupling ratios between them, though it can induce small changes in either direction.

Cadmium was applied in 8 preparations of the isolated Gl in which the SGs had been photoinactivated, and the residual LG-FF interaction was apparent. In 2 preparations, there was no discernible change, but in the remaining 6 , there was a definite, and sometimes dramatic, decrease in the size of the EPSP 
A
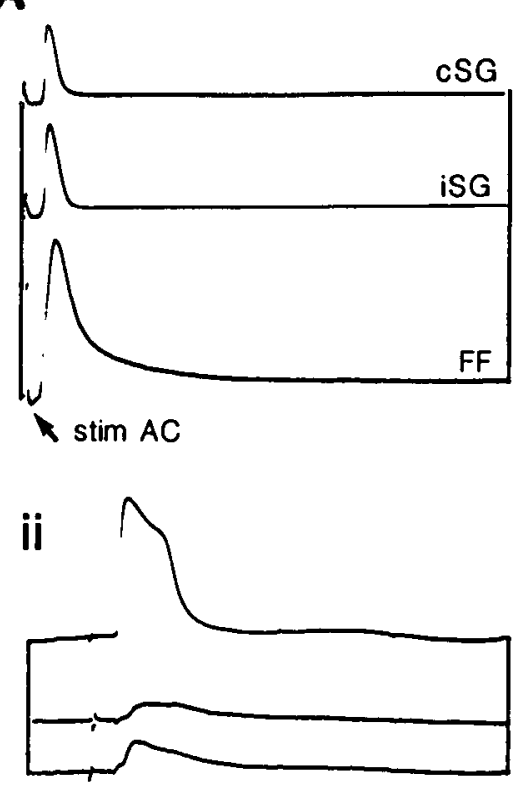

$\mathrm{Bi}$

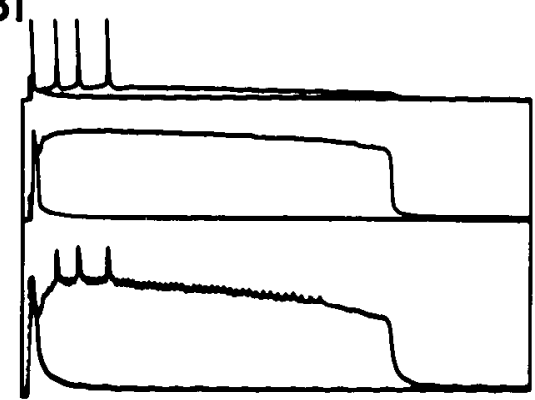

iii

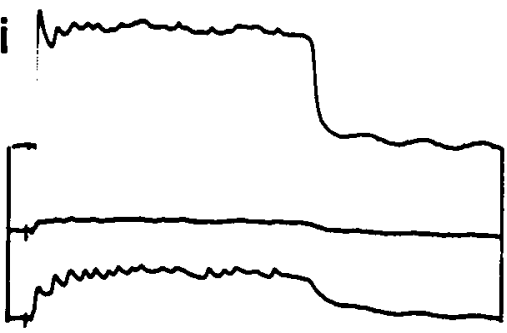

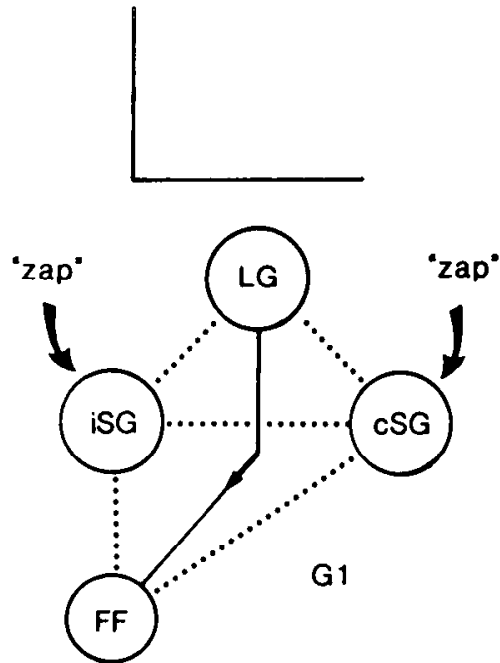

Figure 6. Differential photoinactivation of bilateral SGs. A, Prior to photoinactivation, stimulating the AC induces spikes in the cSG (Ist trace) and iSG (2nd trace), and a large EPSP in the FF ( $3 r d$ trace). B, Differential time course of photoinactivation of the 2 SGs. $i$, The iSG spike has a prolonged plateau, but the cSG spike is still relatively normal. $i i$, The iSG spike has failed, and the cSG spike is starting to broaden. $i i i$, The iSG is almost completely photoinactivated, and the cSG spike has a prolonged plateau. Calibration bars: Vertical $-F F, 30 \mathrm{mV} ; S G(A ; B, i), 150 \mathrm{mV}$; $S G(B, i i), 60 \mathrm{mV} ;$ iSG $(B, i i i), 30 \mathrm{mV} ; c S G(B, i i i), 60 \mathrm{mV}$. Horizontal-A; $B, i i, i i i, 20 \mathrm{msec} ; B, i, 200 \mathrm{msec}$.
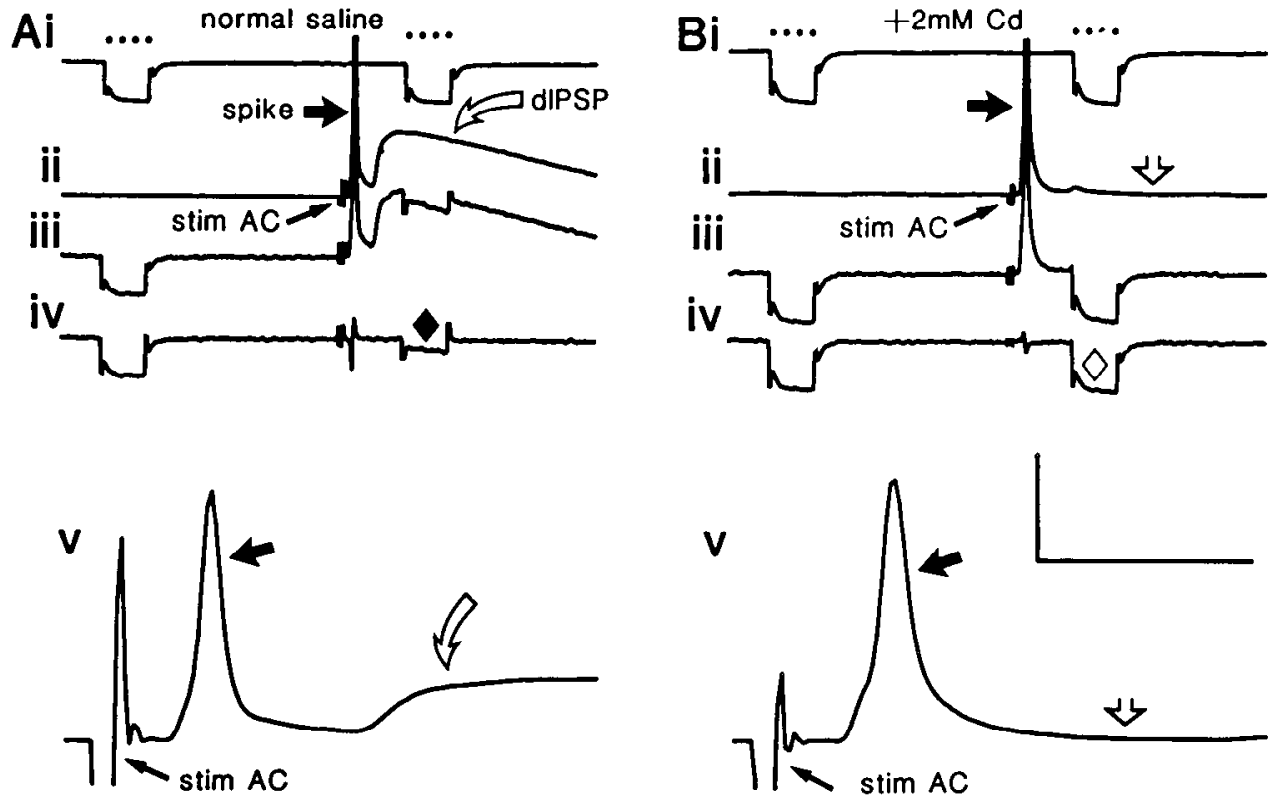

Figure 7. Cadmium ions block chemical transmission but do not block 1:1 spike transmission at GF-MoG synapse. $A$, Normal saline. $i$, Two identical pulses of negative current are injected into the MoG (dotted line over trace), and identical voltage responses are recorded through a second electrode. $i i$, Stimulating the AC elicits a spike in the MoG (solid arrow) followed by a dIPSP (open arrow). iii, The stimulus regimes of $i$ and $i i$ are combined, with the second current pulse timed to coincide with the dIPSP. $i v$, The record in $i i$ has been digitally subtracted from the record in iii. Note the large decrease in the voltage response to the second pulse of current injection, indicating a decrease in membrane impedance during the dIPSP (solid diamond). $v$, The same record as in $i$ at an expanded timebase. $B$, Saline containing 2 mu cadmium ions. $i$, In saline containing cadmium, 2 identical pulses of negative current still elicit identical voltage responses. $i i$, In saline containing cadmium, stimulating the AC still elicits a spike in the MoG (solid arrow), but there is no following dIPSP (open arrow). iii, Same as in $A$, iii, but in cadmium saline. iv, Same as in $A$, $i v$. Note that there is now no decrease in the voltage response to the second current pulse (open diamond), indicating that cadmium ions block the conductance increase as well as the voltage response of the dIPSP. $v$, The same record as in $i i$ at an expanded timebase. Calibration bars: Vertical $-26 \mathrm{mV}$. Horizontal $-A$ and $B, i-i v, 40 \mathrm{msec}, A$ and $B, v, 4 \mathrm{msec}$. 

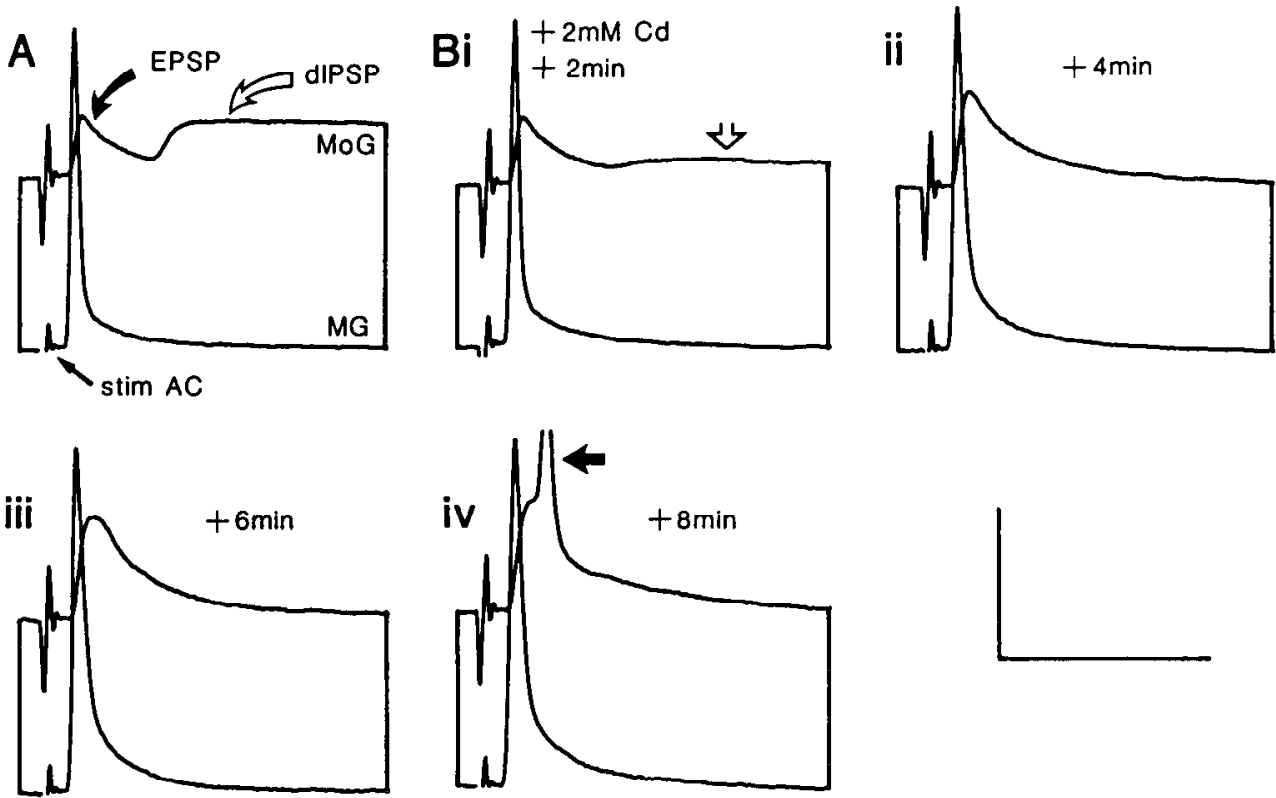

Figure 8. The effects of cadmium ions on subthreshold transmission at MGMoG synapse. $A$, In normal saline, $\mathrm{AC}$ stimulation initiates an EPSP (solid curved arrow) and dIPSP (open arrow) in the fourth MoG (1st trace), and a spike in left fourth MG (2nd trace). $B$, $i-i v$, Two mu cadmium was added to the saline while maintaining AC stimulation at $0.5 \mathrm{~Hz}$. Records are at 2 -min intervals. The dIPSP is rapidly abolished (open arrow), and this is followed by broadening of the presynaptic MG spike and an increase in EPSP amplitude. In $i v$, the EPSP becomes suprathreshold (arrow). Calibration bars: vertical, $40 \mathrm{mV}$; horizontal, $20 \mathrm{msec}$. recorded in the FF when the LG was induced to spike with injected current (Fig. 9A,B). The reduccd EPSP no longer showed the decrement with repeated $L G$ activation that occurred in normal saline. Extracellular stimulation of the third root (R3) showed no change in the amplitude of the antidromic FF spike, but there was an increase in its duration.

\section{Effects of cooling}

The SGs were dye-filled and photoinactivated as usual, then 2 microelectrodes were placed in the LG, and one in an FF. The LG-FF interaction was measured at room temperature (about $20^{\circ} \mathrm{C}$; Fig. $10 A$ ). Cold saline was then superfused into the preparation bath to take it down to a tempcrature of about $7^{\circ} \mathrm{C}$. The duration of the LG spike increased as the temperature decreased, as would be expected (Hodgkin and Huxley, 1952). However, the total amplitude of the EPSP decreased (the opposite of what would be expected with an electrical synapse given the increased duration of presynaptic spike), and 2 distinct components became visible. The initial component of the EPSP still had a very short latency, but there was now a second, longer-latency component (Fig. 10B). The preparation was allowed to recover to room temperature, then saline containing 2 mM cadmium was
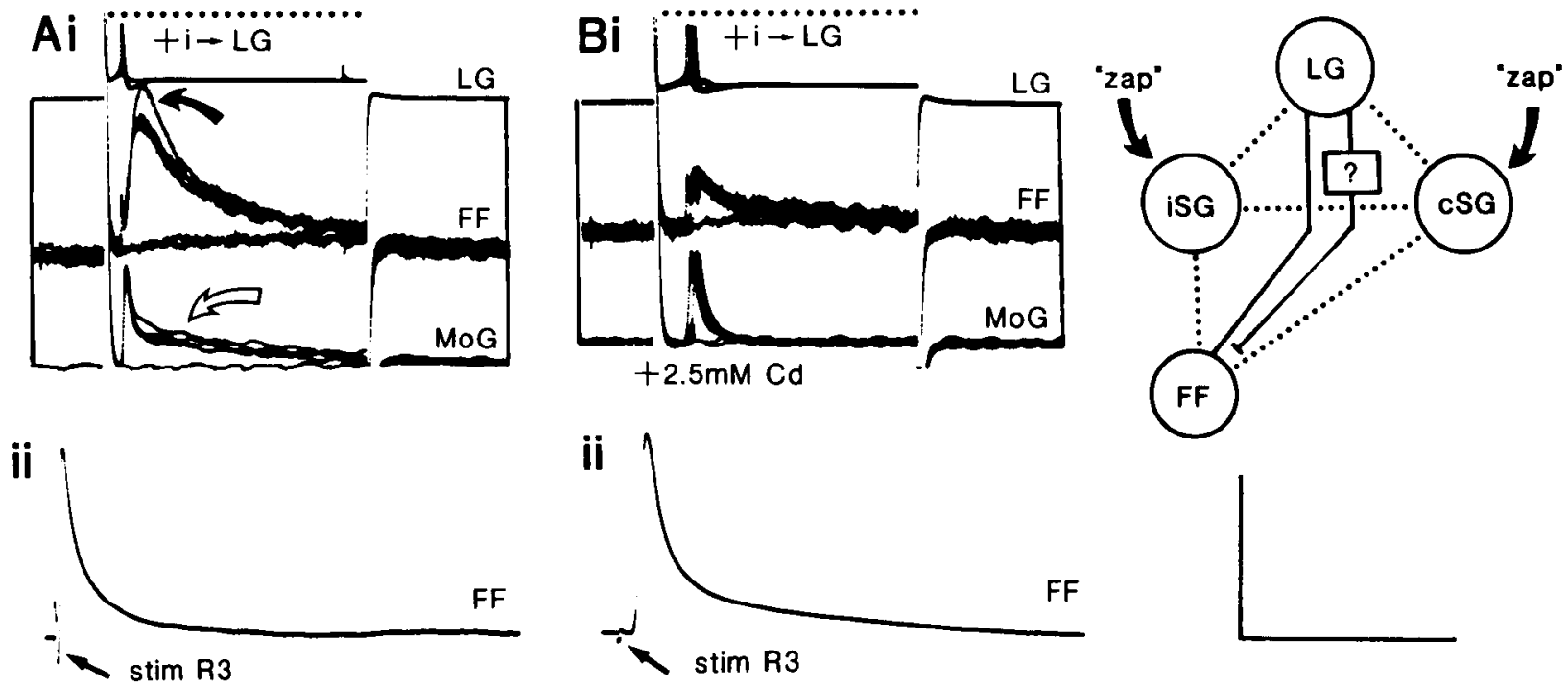

Figure 9. Effects of cadmium on direct LG-FF connection in G1 after bilateral SG photoinactivation. $A$, Normal saline. $i$, Superimposed records (dotted line) show positive current pulses injected into the LG (1st trace) at $1 \mathrm{~Hz}$. The first 2 pulses were just subthreshold. The stimulus amplitude was then increased slightly so that the remaining pulses elicited LG spikes. The first LG spike initiated a large EPSP in the FAC FF (2nd trace, solid arrow) followed by smaller EPSPs, and an EPSP of constant amplitude in the MoG (3rd trace). Note dIPSP in the MoG (open arrow). $i i$, Stimulating R3 of the next anterior ganglion (the last thoracic) elicits an antidromic spike in the FF. $B$, Saline containing 2.5 mM cadmium ions. $i$, Records as in $A, i$. Note the constant decreased amplitude of EPSP in FF and the loss of dIPSP in the MoG. $i i$, Same as in $A$, $i i$. Note the broadening of the antidromic spike. Calibration bars: Vertical $-L G(i), 150 \mathrm{mV} ; F F(i), 6 \mathrm{mV} ; M o G(i), 15 \mathrm{mV} ;$ ii, all traces, $60 \mathrm{mV}$. Horizontal, 20 msec. 

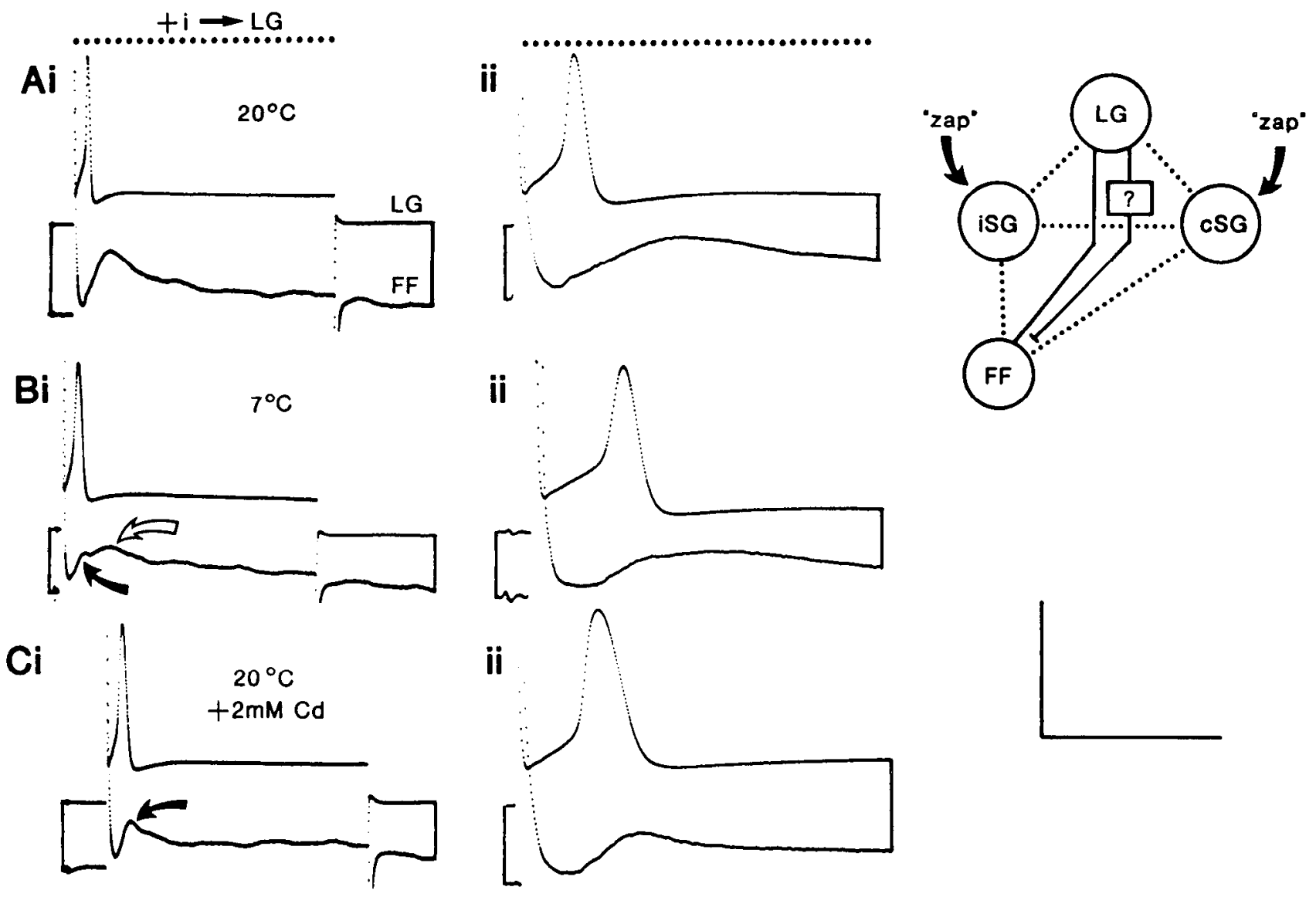

Figure 10. Effects of cooling compared to effects of cadmium on LG-FF synapse following bilateral SG photoinactivation. $A, i$. In normal saline at room temperature, a pulse of current (dotted line) injected into the LG (1st trace) elicits an LG spike and an EPSP in the FPI FF (2nd trace). $A$, $i i$, A similar record with an expanded timebase. $B, i$, Same as in $A, i$, with the preparation cooled to approximately $7^{\circ} \mathrm{C}$. A discontinuity between a short-latency EPSP (solid arrow) and a longer-latency component (open arrow) is now clearly apparent. $B$, $i i$, A similar record at an expanded timebase. Note that the LG spike is broader but slightly smaller than at room temperature. $C, i$, In saline containing 2 mM cadmium, the LG spike elicits a large short-latency EPSP (arrow), but the longer-latency component is missing. $C, i i$, A similar record with an expanded timebase. Note that the LG spike is broader and has increased amplitude compared to the spike in normal saline. Calibration bars: Vertical-LG, $60 \mathrm{mV} ; F F, 15$ $\mathrm{mV}$. Horizontal-i, $20 \mathrm{msec} ; i i, 4 \mathrm{msec}$.

superfused into the preparation bath. This also increased the duration of the LG spike, and produced a short-latency EPSP in the FF that was smaller in amplitude and shorter in duration than the control EPSP, but slightly larger than the EPSP occurring at low temperature (Fig. 10C).

\section{Discussion}

The importance of evolution in understanding neural circuits The study of neural circuits sometimes reveals the presence of seemingly redundant, inefficient, or even maladaptive elements. In the past, the tendency has been to assume that this merely reflected our failure to appreciate all aspects of the true function of that circuit, and indeed, it is hard to prove that this is not so in any individual case. However, there is a growing appreciation that the explanation of many apparent circuit anomalies may lie not in some speculative and obscure functional requirement, but rather in the evolutionary history by which the circuit has achieved its modern form (Dumont and Robertson, 1986; Siegler, 1987). The characteristics of animals, including the circuitry of their nervous systems, are essentially contingent; they arise through the process of natural selection, fueled by random changes in the genetic material. The "... primary criterion of order in the domain of contingency is, and must be, chronology" (Gould, 1990). Thus, in order to understand why a particular circuit operates the way that it does, we have to understand the evolutionary history of that circuit.

To date, the evolutionary explanation has only been invoked for rather minor anomalies within a circuit. However, it is possible that a very major element within the crayfish escape circuit, to whit, the presence of the SG neuron, has its explanation in the evolutionary history of the animal (Heitler and Fraser, 1986). By removing this evolutionarily advanced layer of the circuit, we have revealed details that are functionally "buried" in the intact animal. Of course, the reduced circuit does not itself represent an earlier evolutionary form; the exposed GF-FF connections are not geological strata, frozen in time beneath the dominant GF-SG-FF circuit. However, the reduced circuit does contain information that sheds light upon the history of the modern circuit, and which theories regarding that history have to take into account.

\section{The pattern of $G F-F F$ connectivity}

There is a frequent tendency in the literature to conflate the properties of the 2 sets of GFs, and this is not surprising given 
the similarities in their output. In the intact animal, the SGs provide a common path by which GF excitation is passed to the FFs in each ganglion, thus ensuring that there is no significant difference between the effect of the 2 types of GF on the FFs. However, photoinactivation of the SGs shows that there are considerable differences between the GFs with respect to their direct interactions with the FFs, and also between the outputs of a single GF in different segments. The MGs make almost no direct output to the FFs; virtually the entire effect of the MG neurons on the FFs is mediated by the SG. In contrast, the LGs make output to the FFs that is a direct, albeit weaker, image of the SG-FF interactions, including an anterior-to-posterior segmental gradient.

These findings lend weight to the suggestion that the MG and LG command neurons have different evolutionary origins. We have previously speculated that the earliest form of the MGinitiated escape behavior was a limb-driven event (Heitler and Fraser, 1986), in which the simultaneous protraction of the segmental appendages, driven by neurons that included a protoSG motorneuron, caused the animal to move backwards. Tail flexion would obviously improve the efficiency of such a behavior, and the FF motorneurons could thus have "added on" to an established MG-SG circuit. Our finding that there is minimal direct input from the MG to the FFs certainly fits with this scheme. On the other hand, there is significant direct input from the LGs to the FFs. Does this represent a remnant of an original circuit in which the LGs drove the FFs directly to produce a tail-flip-mediated escape, and into which the SG somehow got interposed, or does it represent the current form of a continually evolving circuit, originally configured as LG-SG-FF, but in which the SG is in the process of being bypassed, because it is now redundant? One may even argue that this bypassing process has itself become redundant, because the massive enlargement of a single FF motorneuron, the MoG, and the evolution of the powerful, direct GF-MoG connection, has removed most of the selective pressure from the GF-SG-FF circuit.

\section{Characteristics of photoinactivation}

The most spectacular feature of the photoinactivation process is the remarkable increase in spike duration shown by the $S G$. Within a few minutes of the start of photoinactivation, the SG spike consistently developed a depolarized plateau of $100-400$ msec duration. Spike broadening during photoinactivation was reported in cockroach axons (Libersat et al., 1989), but the maximum duration of the spike in that system was less than 10 msec, while there is no report of spike broadening at all in cricket auditory interneurons (Selverston et al., 1985). At the stage in photoinactivation when the SG shows this broadened spike, it still has a substantial resting membrane potential, and the electrical synapses to the FFs and contralateral SG are still functional. Consequently, the broadened SG spike results in a massive increase in the amplitude of the EPSP in these neurons. There is no back-propagation of the sustained depolarization to the GFs, indicating not only that the electrical synapses are still operating, but that the rather more labile rectification mechanism (Jaslove and Brink, 1986) is also still intact.

The spike broadening suggests that the first victim of the photoinactivation process is an intracellular moiety concerned with the restitution of the membrane potential following a spike. Obvious candidates are the inactivation gates of the sodium channels, or the $\mathrm{Ca}^{2+}$-activated potassium channels. We have not attempted to "freeze" a neuron in the partially inactivated state with prolonged action potential, but if this were possible, it might prove a useful tool for investigating these cellular mechanisms.

Eventually, the SG spike starts to fail, and its membrane potential decreases. At this stage, the electrical synapses must start to block, because we did not observe a significant decrease in the resting potential of the postsynaptic neurons. When the SGs had completely lost all membrane potential and showed no response to stimulation of the GFs, they were regarded as dead. At this time, the postsynaptic neurons showed no significant change in resting potential compared to their initial values prior to photoinactivation, nor did the amplitude of their antidromic spikes change. At no point did the GFs presynaptic to the SGs show any change in response to the photoinactivation process. We thus conclude that only the SGs themselves were effected by the experimental procedure.

It took 30-60 min to photoinactivate the SGs, which is rather longer than that reported for most other preparations (e.g., Selverston et al., 1985; Libersat et al., 1989). This is probably because of the very large size of the SG; we find that small local interneurons in the crayfish can be photoinactivated within 5 min (K. Fraser and W.J. Heitler, unpublished observations).

\section{Dual-component GF-FF transmission}

One of the striking characteristics of the crayfish GFs evident from the physiological literature is that their entire known output is mediated by electrical synapses. Consistent with this, the direct connection between the LG and FFs also has a major electrical component. However, an unexpected finding of this project was that the direct LG-FF connection also contains a chemical component. The main evidence for this comes from applying cadmium ions to block calcium channels in the presynaptic terminals (and elsewhere, of course) and thus block transmitter release. The effectiveness of this procedure was demonstrated in control experiments in which $2 \mathrm{mM}$ cadmium totally blocked known chemical PSPs and their associated postsynaptic conductance change, but actually enhanced known electrical PSPs, probably due to broadening of the presynaptic spike. When cadmium was applied to a preparation in which the SGs had been photoinactivated, it usually produced a marked decrement in the amplitude of the EPSP elicited in an FF in response to a GF spike, thus suggesting that there was a chemical component. Further evidence for a chemical component comes from cooling the preparation. This induced a 2-phase EPSP, with an initial short-latency component, and a second, delayed component, which was reduced in amplitude compared to the control. Because cooling the preparation caused the presynaptic GF spike to broaden, these results again suggest that the firstphase response is an electrical EPSP, while the second-phase response is a chemical EPSP.

It is difficult to prove conclusively that the chemical component results from a monosynaptic output from the GF. There is no known pathway other than the SGs mediating excitation from the GFs to the FFs, but we cannot rule out the possibility that an unidentified path exists. We have encountered a variety of unidentified interneurons that are driven by the GFs, and it is possible that some such neuron might make chemical output to the FFs. However, in the majority of cases, these interneurons appeared to receive their inputs from the GFs via the SGs (like the identified CDIs I2 and I3), and so could not be the source of the FF chemical input. The latency of the chemical component is short, because there was frequently no discontinuity 
visible in the rising phase of the EPSP; the chemical component simply extended the peak of the electrical component. Even when a discontinuity was evident, it was never very pronounced at room temperature. However, we could not measure the chemical latency accurately, because of its superimposition upon the electrical component. We know of no method whereby the electrical component could be removed without also substantially altering the chemical component. If the interaction is indeed monosynaptic, it is the first time a functional chemical output has been identified from a crayfish GF.

\section{Structural and functional comparison of GF-FF and GF-MoG transmission}

There has only been one, rather brief, EM study of the GF-FF contacts, and this found no evidence for chemical synapses (Atwood and Pomeranz, 1974). However, not all appositions were examined, and therefore the presence of a small chemical component was not completely excluded. In contrast, there have been several detailed studies on the ultrastructure of the GFMoG contacts. The MoG is generally regarded as having evolved from an ordinary FF motorneuron, and therefore a comparison is of interest. The dominant features of the GF-MoG appositional regions are the large and prominent plaques of gap junctions (e.g., Hanna et al., 1978), which correlate very well with the powerful electrical synapse connecting the 2 neurons (Furshpan and Potter, 1959a). There is no physiological evidence for direct chemical transmission at the GF-MoG synapse (e.g., Figs. 7, 8), but in the crayfish Procambarus, there are nonetheless structures that look like chemical synapses occurring in parallel to the gap junctions (Krasne and Stirling, 1972). In Pacifastacus (the species of the present study), there are few, if any, chemical profiles at the GF-MoG synapse in adults, but such profiles are very common in juveniles. Indeed, chemicallike profiles are the earliest detectable form of specialized junctional apposition, with the gap junction plaques gradually appearing in the midst of such profiles (Leitch et al., 1989). However, even in very young crayfish, there is no physiological evidence for chemical transmission, while rectifying electrical transmission is clearly present from the day of hatching onwards (Heitler et al., 1990). It is difficult to make sense of these apparently contradictory findings, but we may speculate that the mixed chemical-electrical transmission of the GF-FF synapses represents an early form of the GF output, and that, as the MoG evolved its specialized form and function, the electrical component became more and more dominant. The chemicallike profiles found at the modern GF-MoG synapse, particularly in the juvenile animals, may be a nonfunctional (in terms of transmission) remnant of the functional chemical synapses still found at the GFFF synapse. Perhaps the chemicallike profiles at the juvenile GF-MoG synapse play a structural and/or developmental role in the early life of the animal.

\section{References}

Atwood HL, Pomeranz B (1974) Crustacean motor neuron connections traced by back filling for electron microscopy. J Cell Biol 63: 329-334.

Dumont JPC, Robertson RM (1986) Neuronal circuits: an evolutionary perspective. Science 233:849-853.

Fraser K, Heitler WJ (1987) Interactions of the giant fibres and motor giant neurones of the hermit crab. J Exp Biol 133:353-370.

Fraser K, Heitler WJ (1989) Thoracic output of crayfish giant fibres. II. The segmental giant neurone. J Comp Physiol A 166:125-132.
Furshpan EJ, Potter DD (1959a) Transmission at the giant motor synapses of the crayfish. J Physiol (Lond) 145:289-325.

Furshpan EJ, Potter DD (1959b) Slow post-synaptic potentials recorded from the giant motor fibre of the crayfish. J Physiol (Lond) 145:326-335.

Gould SJ (1990) Wonderful life, p 15. London: Hutchinson Radius.

Hanna RB, Keeter JS, Pappas GD (1978) The fine structure of a rectifying electrotonic synapse. J Cell Biol 79:764-773.

Heitler WJ, Darrig S (1986) The segmental giant neurone of the signal crayfish, and its interactions with abdominal fast flexor and swimmeret motorneurones. J Exp Biol 121:55-75.

Heitler WJ, Fraser K (1986) The segmental giant neurone of the hermit crab, Eupagurus bernhardus. J Exp Biol 125:245-269.

Heitler WJ, Fraser K (1989) In situ dye-visualization and photoinactivation using an epifluorescent compound microscope. J Exp Biol 145:477-481.

Heitler WJ, Leitch BM, Pitman R.M, Cobb JLS (1990) The postembryonic development of rectifying electrical synapses in the crayfish: physiology. J Neurocytol, in press.

Hodgkin AL, Huxley AF (1952) A quantitative description of membrane current and its application to conduction and excitation in nerves. J Physiol (Lond) 117:500-544.

Jaslove SW, Brink PR (1986) The mechanism of rectification at the electrotonic motor giant synapse of the crayfish. Nature 323:63-65.

Kostyuk PG, Krishtal OA, Shakhovalov YA (1977) Separation of sodium and calcium currents in the somatic membrane of mollusc neurones. J Physiol (Lond) 270:545-568.

Kramer AP, Krasne FB, Wine JJ (1981) Interneurons between giant axons and motoneurons in crayfish escape circuitry. J Neurophysiol 45:550-573.

Krasne FB, Stirling CA (1972) Synapses of crayfish abdominal ganglia with special attention to afferent and efferent connections of the lateral giant fibers. Z Zellforsch Mikrosk Anat 127:526-544.

Leitch B, Heitler WJ, Cobb JLS, Pitman RM (1989) Post-embryonic development of rectifying electrical synapses in the crayfish: ultrastructure. J Neurocytol 18:749-761.

Leitch B, Heitler WJ, Cobb JLS, Pitman RM (1990) Anti-GABA antibodies label a sub-population of chemical synapses which modulate an electrical synapse in crayfish. J Neurocytol, in press.

Libersat F, Selverston A, Camhi JM, Goldstein RS (1989) Photoinactivation of a portion of a neurone for long-term studies of its role in behaviour. J Exp Biol 142:453-459.

Miller JP, Selverston AJ (1979) Rapid killing of single neurons by irradiation of intracellularly injected dyes. Science 206:702-704.

Miller LA, Hagiwara G, Wine JJ (1985) Segmental differences in pathways between crayfish giant axons and fast flexor motoneurons. $\mathrm{J}$ Neurophysiol 53:252-265.

Mittenthal JE, Wine JJ (1978a) Connectivity patterns of crayfish giant interneurons: visualization of synaptic regions with cobalt dye. Science 179:182-184.

Mittenthal JE, Wine JJ (1978b) Segmental homology and variation in flexor motoneurons of the crayfish abdomen. J Comp Neurol 177: 311-334.

Ochi R (1969) Ionic mechanism of the inhibitory post-synaptic potential of crayfish motor giant fiber. Eur J Physiol 311:131-143.

Purves RD (1981) Microelectrode methods for intracellular recording and ionophoresis. London: Academic.

Roberts A, Krasne FB, Hagiwara G, Wine JJ, Kramer AP (1982) Segmental giant: evidence for a driver neuron interposed between command and motor neurons in the crayfish escape system. J Neurophysiol 47:761-781.

Selverston AI, Remler MP (1972) Neural geometry and activation of crayfish fast flexor motoneurons. J Neurophysiol 35:797-814.

Selverston AI, Kleindienst H-U, Huber F (1985) Synaptic connectivity between cricket auditory interneurons as studied by selective photoinactivation. J Neurosci 5:1283-1292.

Siegler MVS (1987) An eye on ancient circuits. Nature 326:130-131.

Stewart WW (1978) Functional connections between cells as revealed by dye-coupling with a highly fluorescent naphthalimide tracer. Cell 14:741-749.

Stirling A (1972) The ultrastructure of giant fibre and serial synapses in crayfish. Z Zellforsch Mikrosk Anat 131:31-45.

Wiersma CAG (1947) Giant nerve fiber system of the crayfish. A contribution to comparative physiology of synapse. J Neurophysiol 10:23-38. 
Wine JJ (1977) Neuronal organization of crayfish escape behavior: inhibition of giant motoneuron via a disynaptic pathway from other motoneurons. J Neurophysiol 40:1078-1097.

Wine JJ (1984) The structural basis of an innate behavioural pattern. J Exp Biol 112:283-319.
Wine JJ, Krasne FB (1982) The cellular organization of the crayfish escape behaviour. In: The biology of crustacea, Vol III, Neural integration (Atwood H, Sandeman D, eds), Ch 15. New York: Academic. 